\title{
Geometrical frustration effects on charge-driven quantum phase transitions
}

\author{
L. Cano-Cortés, ${ }^{1}$ A. Ralko, ${ }^{2}$ C. Février, ${ }^{2}$ J. Merino, ${ }^{1}$ and S. Fratini ${ }^{2}$ \\ ${ }^{1}$ Departamento de Física Teórica de la Materia Condensada,Universidad Autónoma de Madrid, E-28049 Madrid, Spain \\ ${ }^{2}$ Institut Néel-CNRS and Université Joseph Fourier, Boîte Postale 166, F-38042 Grenoble Cedex 9, France
}

(Received 24 June 2011; published 14 October 2011)

\begin{abstract}
The interplay of Coulomb repulsion and geometrical frustration on charge-driven quantum phase transitions is explored. The ground-state phase diagram of an extended Hubbard model on an anisotropic triangular lattice relevant to quarter-filled layered organic materials contains homogeneous metal, "pinball," and threefold charge ordered metallic phases. The stability of the pinball phase occurring for strong Coulomb repulsions is found to be strongly influenced by geometrical frustration. A comparison with a spinless model reproduces the transition from the homogeneous-metallic phase to a pinball liquid, which indicates that the spin correlations should play a much smaller role than the charge correlations in the metallic phase close to the charge-ordering transition. Spin degeneracy is, however, essential to describe the dependence of the system on geometrical frustration. Based on finite-temperature Lanczos diagonalization we find that the effective Fermi temperature scale $T^{*}$ of the homogeneous metal vanishes at the quantum phase transition to the ordered metallic phase driven by the Coulomb repulsion. Above this temperature scale "bad" metallic behavior is found which is robust against geometrical frustration in general. Quantum critical phenomena are not found whenever nesting of the Fermi surface is strong, possibly indicating a first-order transition instead. "Reentrant" behavior in the phase diagram is encountered whenever the $2 k_{F}$ charge-density wave instability competes with the Coulomb driven threefold charge order transition. The relevance of our results to the family of quarter-filled materials, $\theta$-(BEDT-TTF) $)_{2}$, is discussed.
\end{abstract}

\section{INTRODUCTION}

Strongly correlated electron materials are often characterized by complex phase diagrams, reflecting an intricate interplay between magnetic, orbital, lattice, and charge degrees of freedom. As these excitations couple to the conduction electrons, the metallic state expected in the absence of interactions has to compete with several ordered phases. Examples of these materials include cuprate superconductors, nickelates, heavy-fermion compounds, transition-metal dichalchogenides, organic charge transfer salts, and the ironbased pnicitide superconductors, all presenting various forms of magnetic, orbital, and charge order. Even when a metallic phase is stabilized, these systems are generally found to exhibit large effective mass enhancements and electrical resistivities violating the Ioffe-Regel-Mott (IRM) condition. ${ }^{1-6}$ Surprisingly enough, such "bad" metallic behavior does not impede the emergence of superconductivity, but rather appears to be a prerequisite for the achievement of high critical temperatures. ${ }^{7}$

Charge-ordered (CO) phases are commonly observed in the class of two-dimensional organic compounds $\theta-\mathrm{ET}_{2} \mathrm{X} \quad(\mathrm{ET}=$ BEDT-TTF, bisethylenedithiotetrafulvalene $)^{8-12}$ and ascribed to the prominent role of electron-electron interactions. ${ }^{13}$ At the noninteracting level, these compounds are predicted to be metals with 3/4-filled electronic bands. The observation of electronic ordering implies that the magnitude of electron-electron interactions is comparable with the widths of the relevant electronic bands constructed from the $\pi$ molecular orbitals. In turn, the presence of such strong interactions raises questions about the nature of the metallic phase in these materials, that should exhibit distinctive features of "correlated electron systems" in the Mott sense. The proximity to charge-ordering instabilities, with the possible emergence of quantum critical points as the transition temperature is made to vanish, is also expected to strongly alter the physical properties of the metal. All these ingredients should lead to measurable deviations from the usual Fermi-liquid behavior, ${ }^{14,15}$ in close analogy with heavy-fermion systems. ${ }^{16-19}$

The minimal theoretical description of the electronic properties of $\theta-\mathrm{ET}_{2} \mathrm{X}$ organic conductors is based on the twodimensional extended Hubbard model (EHM) on a triangular lattice. Several theoretical studies have aimed at reproducing the different $\mathrm{CO}$ patterns realized in this class of materials, either within the framework of the EHM itself or its generalizations, including longer ranged electronic interactions and various types of electron-lattice interactions. ${ }^{20,21}$ In the present work we focus on the following open issues: (i) How does the strength of the local Coulomb correlations modify the nature of the metallic phase as well as its CO instabilities? (ii) What are the effects of geometrical frustration in the electron motion arising from the triangular molecular arrangement? (iii) How does the proximity to a given $\mathrm{CO}$ phase extend its influence onto the properties of the correlated metal, possibly leading to non-Fermi-liquid behavior?

In Sec. II we set the minimal electronic model needed for the study of electronic properties of $\theta-(\mathrm{ET})_{2} \mathrm{X}$ compounds and provide a brief review of established theoretical results. In Sec. III the model is solved by Lanczos diagonalization and the zerotemperature phase diagram is obtained for different degrees of geometrical frustration. In Sec. IV the resulting metallic phases are explored through a finite- $T$ Lanczos diagonalization calculation. ${ }^{22-24}$ We theoretically explore the consequences of a quantum critical point (QCP) at a charge-ordering transition driven by the quantum fluctuations associated with strong intersite Coulomb repulsion. Our results are compared with a spinless calculation in order to assess the importance of the magnetic degrees of freedom in the observed quantum 
criticality and make contact with the existing literature. The relevance of the present results to the physics of $\theta$-type ET compounds is discussed in the conclusive Sec. V.

\section{MODEL AND METHOD}

\section{A. Extended Hubbard model on the triangular lattice}

Quasi-two-dimensional organic conductors (general formula $A_{2} B$ ) are charge transfer compounds composed of alternating layers of conducting (donor) molecules $A$ and insulating (acceptor) units $B$. They exhibit a large variety of molecular arrangements corresponding to different polytypes classified by greek characters. ${ }^{13,25}$ The materials of the $\theta-\mathrm{ET}_{2} \mathrm{X}$ class have a triangular lattice structure, shown in Fig. 1, with an average of $n=3 / 2$ electrons per molecule, fixed by complete charge transfer between $A$ and $B$ units. Since this corresponds to a three-quarter filled electronic band, these materials should be normal metals in the absence of interactions.

The electronic properties of $\theta-\mathrm{ET}_{2} \mathrm{X}$ materials are commonly described via the extended Hubbard model (EHM),

$$
\begin{aligned}
H= & -t_{p} \sum_{\langle i j\rangle_{p} \sigma}\left(c_{i \sigma}^{\dagger} c_{j \sigma}+\text { H.c. }\right)-t_{c} \sum_{\langle i j\rangle_{c} \sigma}\left(c_{i \sigma}^{\dagger} c_{j \sigma}+\text { H.c. }\right) \\
& +U \sum_{i} n_{i \uparrow} n_{i \downarrow}+V_{p} \sum_{\langle i j\rangle_{p}} n_{i} n_{j}+V_{c} \sum_{\langle i j\rangle_{c}} n_{i} n_{j} .
\end{aligned}
$$

This model includes transfer integrals between the $\pi$ orbitals of nearest-neighboring molecules in the conducting plane, labeled by $t_{p}$ and $t_{c}$ according to the bond directions, a local (on-site) Coulomb repulsion energy $U$, and nonlocal (nearest-neighbor) repulsion terms $V_{c}, V_{p}$ (see Fig. 1 ). ${ }^{26}$

Early mean-field calculations ${ }^{27-29}$ indicated that three types of striped patterns (vertical, diagonal, and horizontal) are realized depending on the relative magnitude of the nonlocal Coulomb interaction parameters. These results were later confirmed by more advanced numerical techniques that can properly account for electronic correlations, such as exact diagonalization $(E D)^{30,31}$ and density matrix renormalization group (DMRG). ${ }^{32}$

More interesting from our perspective is the isotropically interacting case, $V_{p}=V_{c} \equiv V .^{20,28,29,32-36}$ There, because of the frustration of intermolecular interactions induced by the

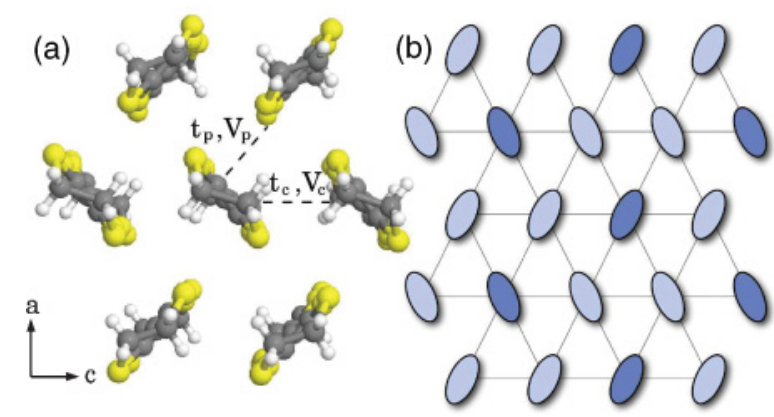

FIG. 1. (Color online) (a) Arrangement of BEDT-TTF molecules in the conducting layers of $\theta$-type ET crystals, with the corresponding transfer integrals and nearest-neighbor Coulomb interactions. (b) Threefold charge ordered phase in the triangular lattice considered in this work. The triangular lattice is defined by the real-space unit vectors $\mathbf{a}_{1}=(1,0)$ defining the $c$ direction and $\mathbf{a}_{2}=(1 / 2, \sqrt{3} / 2)$. triangular lattice geometry, an alternative charge-ordering pattern with threefold periodicity is favored with respect to the (degenerate) striped arrangements, illustrated in Fig. 1(b). A more exotic situation is found in the limit of strong local Coulomb interactions (or, similarly, in a fully spin polarized electron system, i.e., for spinless electrons), where the constraint of no double occupancy on molecular sites converts this threefold order into a partially ordered phase termed "pinball liquid" (PL): 15,32,34,36 This state shows a threesublattice structure with the same symmetry as the threefold phase, in which the carriers of one sublattice are essentially localized as a Wigner-crystal (pin), with the remaining charges (balls) forming an itinerant liquid on the interstitials. It is not clear at present how the transition between these two qualitatively different forms of threefold order takes place as a function of the local Coulomb repulsion $U$. This issue will therefore be thoroughly discussed here.

In addition to the effects of the local electronic correlations, we are interested in the effects of geometrical frustration in the electronic motion, which are triggered by the strongly directional $\pi$ overlaps between neighboring molecules. ${ }^{37}$ This issue is of particular importance to actual materials, as the relative values of the transfer integrals $t_{c}$ and $t_{p}$ can be tuned experimentally by applying pressure or by chemical substitution, which modifies the relative angles between neighboring molecules. ${ }^{38,39}$ As a general observation, negative values of the ratio $t_{c} / t_{p}$ produce the highest charge ordering temperatures, ${ }^{20,38}$ while vanishing or positive values lead to glassy $\left[\mathrm{X}=\mathrm{CsCo}(\mathrm{SCN})_{4}, \mathrm{X}=\mathrm{CsZn}(\mathrm{SCN})_{4}\right]^{40}$ or even superconducting $\left(X=I_{3}\right)$ ground states..$^{20,38,41}$ From a more theoretical point of view, how the system evolves from a perfectly isotropic triangular lattice at $t_{c}= \pm t_{p}$ to a square lattice at $t_{c}=0$ remains an open issue.

Transfer integrals obtained through the Hückel approximation in quarter-filled $\theta$-ET crystals are in the range $-0.5 \lesssim$ $t_{c} / t_{p} \lesssim 1.5$, with $t_{p} \approx-0.05$ to $0.1 \mathrm{eV} .{ }^{39}$ These values generally differ from the ones extracted from optical reflectivity and de Haas-van Alphen experiments ${ }^{41}$ for each specific crystal. On the other hand, Coulomb repulsion energies in organic molecular crystals ${ }^{42}$ have been estimated by calculating the screening corrections to the bare repulsion energies of the isolated molecules, $U_{0}$ and $V_{0}$, obtained from $a b$ initio calculations. ${ }^{43}$ These calculations lead to Hubbard parameters of the model Eq. (1): $U \sim U_{0} / 2 \sim(15-20)\left|t_{p}\right|$, and $V_{p} \sim$ $V_{c} \sim U / 2$, with a bandwidth $W \sim(8-9)\left|t_{p}\right|$. These Coulomb energies are larger than assumed in previous works: ${ }^{31,32,36,44}$ $U \sim(8-10)\left|t_{p}\right|$, and $V \sim(1-3)\left|t_{p}\right|$, as extracted from optical reflectivity measurements. ${ }^{13}$ The degree of uncertainty in the microscopic parameters implies that a general understanding of the model and its phase diagram in the full parameter space $U, V$, and $t_{c} / t_{p}$ is essential. This is the main focus of the present work.

\section{B. Finite-T Lanczos approach}

We perform ED calculations through a finite- $T$ Lanczos algorithm with periodic boundary conditions. ${ }^{22,23}$ The large number of excited states inherent to the many-body problem which are needed to evaluate statistical sums is cut off by keeping only a small number of low-lying states at each 
temperature. This is performed through an Arnoldi algorithm ${ }^{24}$ which reduces the size of the Hilbert space enormously. The accuracy of the method is restricted to temperatures which are not too low, i.e., not lower than the energy of the lowest excitation of the quantum many-body system. For the method to be practical $T$ should not be so large that one needs to keep too many states in the statistical sums. Finite-size effects are somewhat reduced by the effect of temperature and the method is quite reliable for extracting integrated properties. Instead, spectral properties such as optical conductivity and photoemission spectra are prone to large finite-size effects, and will not be analyzed here.

Due to the high computational demand of the finite- $T$ algorithm, the calculations are performed on an $N_{s}=12$ site cluster. In principle, a larger $N_{s}=18$ cluster whose geometry is also suitable for reproducing the threefold $\mathrm{CO}$ pattern could be used at $T=0$. However, we shall not consider such case because a 3/4-filling implies a different number of spin-up and spin-down electrons, while the ground state is expected to be in a $S=S_{z}=0$ state.

We characterize the physical properties of the different phases based on the following quantities, which are accessible through finite- $T$ Lanczos calculations.

(i) Charge correlation function. The charge structure factor signaling the possible occurrence of a charge-ordered state in the system is evaluated at finite- $T$ through

$$
C(\mathbf{q})=\frac{1}{Z} \sum_{m} e^{-\beta E_{m}}\left\langle m\left|\frac{1}{N_{s}^{2}} \sum_{i, j} e^{i \mathbf{q} \cdot \mathbf{R}_{i j}} n_{i} n_{j}\right| m\right\rangle .
$$

Here $Z=\sum_{m} e^{-\beta E_{m}}$ is the partition function of the system and $\beta=1 / k_{B} T$. A charge-ordered state with modulation $\mathbf{Q}$ is signaled if $C(\mathbf{Q})$ is finite in the thermodynamic limit. The threefold ordering corresponds to a charge density modulation with wave vector $\mathbf{Q}=(2 \pi / 3,2 \pi / \sqrt{3})$, which lies at the corner of the hexagonal Brillouin zone, see Fig. 11 (all corners are equivalent, being connected by reciprocal lattice vectors or time-reversal symmetry). An accurate numerical determination of the phase boundaries should rely on a proper finite-size scaling of the results. While this is prohibitive for the fermionic system under study due to the rapidly increasing size of the Hilbert space, the ordering transitions can still be identified as the locus of steepest variation of charge correlations upon varying the microscopic parameters of the model.

(ii) Kinetic energy. This quantity provides direct information on how the motion of the charge carriers is slowed down by interactions. It can be evaluated with high accuracy from the finite- $T$ Lanczos diagonalization, because it results from a quantum mechanical and thermal average over a huge number of states. By normalizing it to a reference noninteracting value $K_{0}$, it gives valuable information on the degree of electronic correlations in the many-body system. ${ }^{45,46}$ Under suitable assumptions, this quantity can be compared with optical absorption experiments in actual materials via the $f$-sum rule. ${ }^{47}$

The kinetic energy is evaluated from the following thermal average,

$$
K=\frac{1}{Z} \sum_{m} e^{-\beta E_{m}}\left\langle m\left|\frac{1}{N_{s}} \sum_{\mathbf{k}, \sigma} \epsilon_{\mathbf{k}} c_{\mathbf{k} \sigma}^{\dagger} c_{\mathbf{k} \sigma}\right| m\right\rangle,
$$

where $|m\rangle$ is the total set of eigenstates of the system with energies $E_{m}$.

(iii) Double occupancy. It is useful to analyze the number of double occupancy per site in the lattice which reads

$$
d=\frac{1}{Z} \sum_{m} e^{-\beta E_{m}}\left\langle m\left|\frac{1}{N_{s}} \sum_{i} n_{i \uparrow} n_{i \downarrow}\right| m\right\rangle,
$$

and is different for the different phases analyzed. For example, it is a key quantity in the analysis of the Mott transition in the half-filled Hubbard model since $d$ is suppressed in the Mott insulator, which allows us to determine the critical Coulomb coupling. In model Eq. (1), $d$ is helpful for characterizing the different possible $\mathrm{CO}$ states for different $U$ and $V$.

(iv) Specific heat. From the total energy of the system, $E=$ $\langle H\rangle$, we can obtain the specific heat by taking the derivative with respect to the temperature $T$ :

$$
C_{V}=\frac{\partial\langle H\rangle}{\partial T} .
$$

Unless otherwise specified, we use units such that $k_{B}=$ $\hbar=1$. The finite-temperature method recovers the groundstate properties by taking the limit $\beta \rightarrow \infty$. In practice this is achieved for $\beta=50-100$ for the various $U$ and $V$ explored across the whole phase diagram. Typically about 30 to 50 terms are kept in the evaluation of the statistical sums over the excited states $|m\rangle$ with corresponding energies $E_{m}$.

\section{PHASE DIAGRAM AT $T=0$}

The zero-temperature phase diagram of the model Eq. (1) in the $(U, V)$ plane is shown in Fig. 2. The phase transition lines are determined using three alternative methods, which all give coincident results: (i) via the evolution of the charge correlation function $C(\mathbf{Q})$ calculated at the threefold wave vector, Fig. 3; (ii) by tracking directly the charge-ordering patterns that develop in real space, Fig. 4; and (iii) by analyzing the fidelity between ground states at different values of the microscopic parameters, Fig. 5, as introduced below.

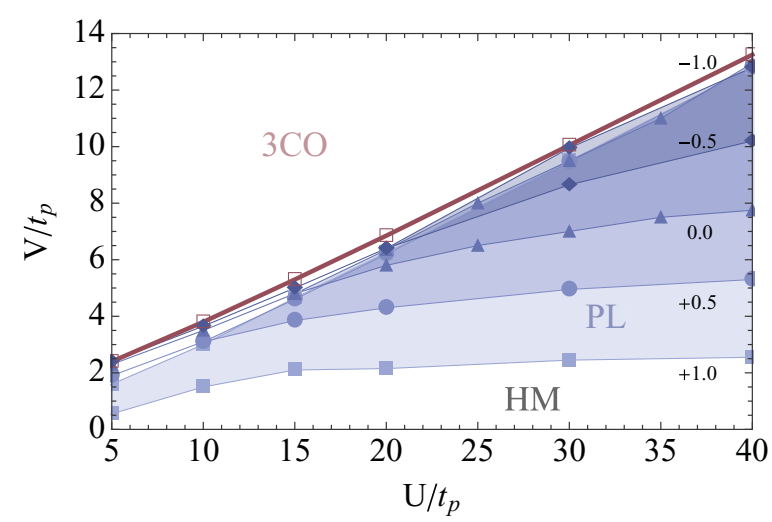

FIG. 2. (Color online) Phase diagram obtained at $T=0$ from numerical diagonalization of a $N_{s}=12$ cluster. The different shaded areas correspond to the pinball (PL) phase for $t_{c} / t_{p}=1,0.5,0,-0.5$ (squares, circles, triangles, diamonds respectively, from light to dark blue). The case $t_{c} / t_{p}=-1$ has no pinball phase in the explored $U$ range. The red line corresponds to a direct transition from the homogeneous-metal (HM) or pinball-liquid (PL) to the threefold charge ordered (3CO) state. 

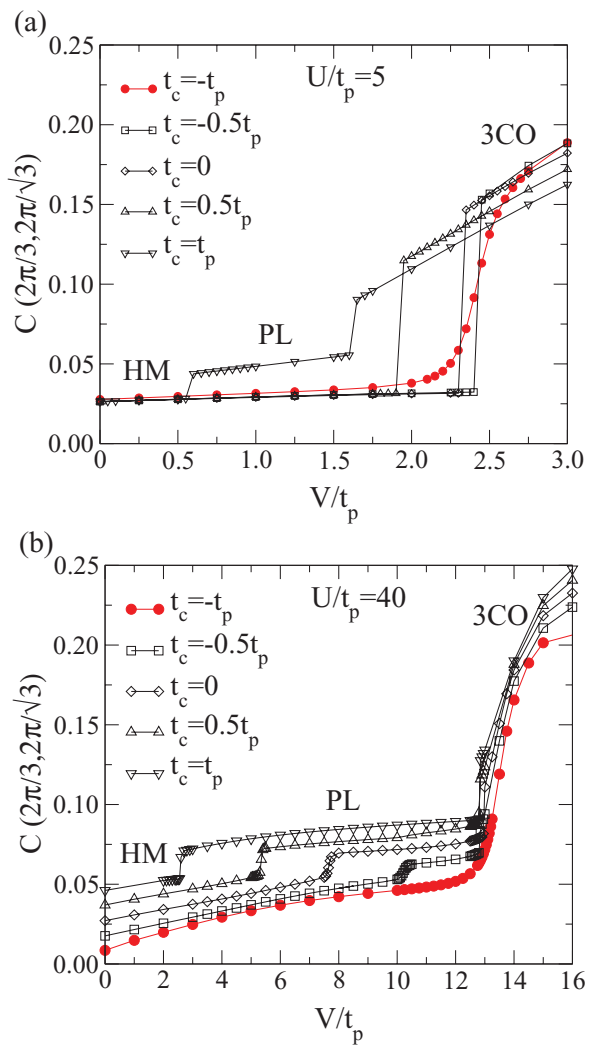

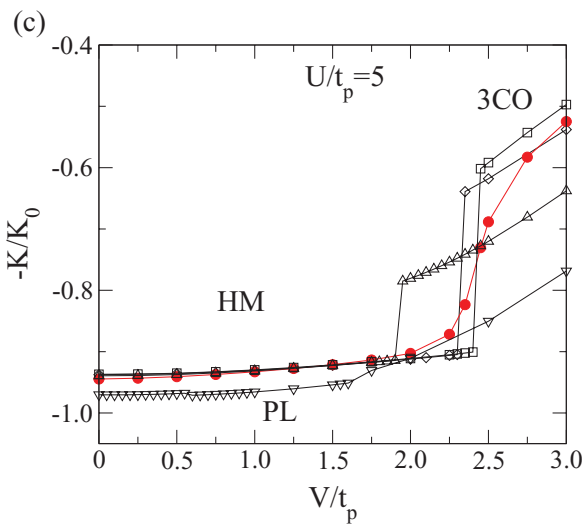

(d)

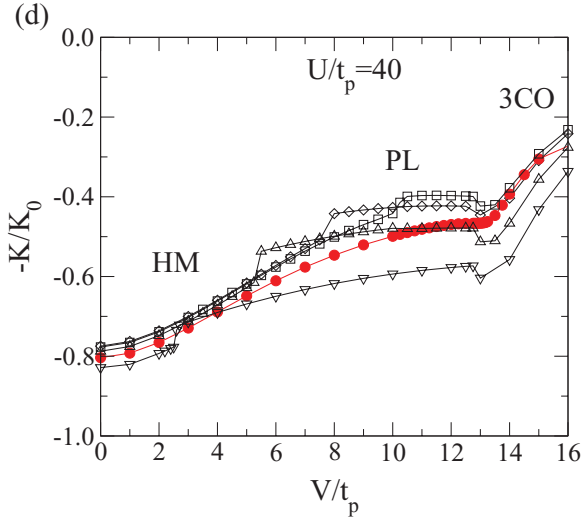

(e)

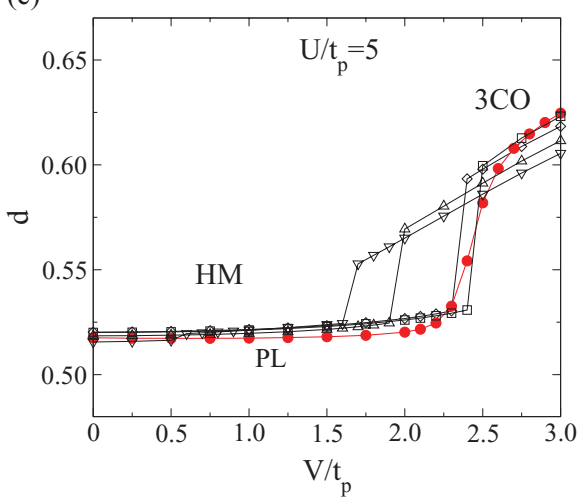

(f)

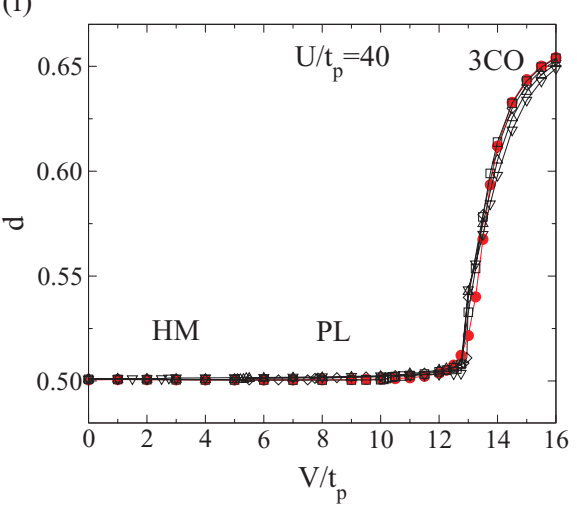

FIG. 3. (Color online) (a), (b) Charge correlation function for the threefold wave vector $\mathbf{Q}=(2 \pi / 3,2 \pi / \sqrt{3})$ as a function of $V / t_{p}$ (a) in the weakly correlated $U=5 t_{p}$ and (b) the strongly correlated limit $U=40 t_{p}$. (c), (d) Average normalized kinetic energy $K / K_{0}$ as a function of $V / t_{p}$. (e), (f) Double-occupancy probability. The plots in (b) have a vertical offset of \pm 0.02 for $t_{c}= \pm t_{p}$ and of \pm 0.01 for $t_{c} \pm 0.5 t_{p}$ for clarity.

We are interested here in the charge-ordering instabilities driven by the intersite repulsion $V$. Our numerical results confirm the existence of three distinct phases: a homogeneous metal (HM) at low $V$, a threefold charge ordered phase (3CO) at large $V$, and an intermediate "pinball-liquid" (PL) phase emerging at large values of $U$. The most striking effect

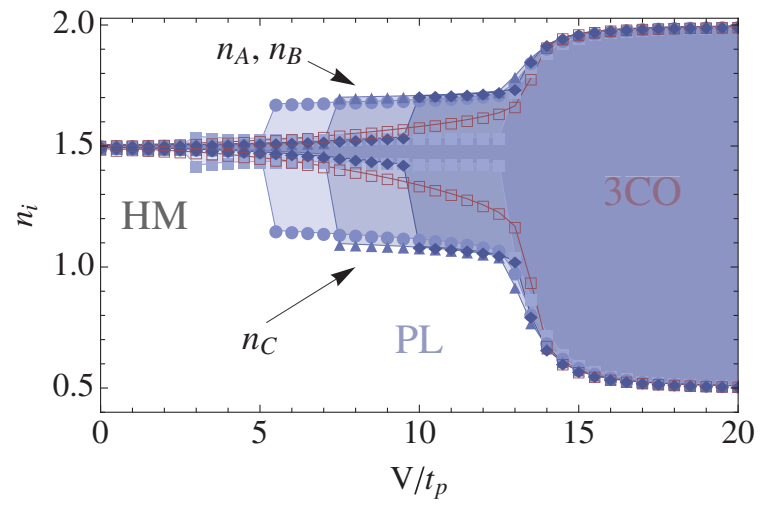

FIG. 4. (Color online) Average electron densities in the electronrich (hole-poor, $n_{A}=n_{B} \geqslant n$, upper curves) and electron-poor (holerich, $n_{C} \leqslant n$, lower curves) sublattices, as obtained from Lanczos diagonalization at $U / t_{p}=40$ in the presence of a weak translational symmetry breaking potential. We take $\delta=+0.05 t_{p}$ on the sites of the hole-rich sublattice in order to select one realization of the threefold symmetry. Color codes are the same as in Fig. 2 (from light to dark blue: $t_{c} / t_{p}=1,0.5,0,-0.5$; red: $t_{c} / t_{p}=-1$ ). in Fig. 2 is that the region of the homogeneous-metallic phase is strongly reduced upon increasing the $t_{c} / t_{p}$ ratio, evidently due to a corresponding stabilization of the competing pinball-liquid phase. We note that the homogeneous metal is always the ground state at $V=0$ independently of the strength of the local repulsion $U$. This can be rationalized by the fact that in the absence of nearest-neighbor interactions, at $n=3 / 2$ the holes $\left(n_{h}=2-n=1 / 2\right)$ can effectively avoid each other when moving along the lattice.

\section{A. Characterization of the different phases}

Figure 3 reports the evolution of the charge correlation function, the kinetic energy, and double occupancy as a function of $V$, along vertical cuts in the phase diagram corresponding to $U / t_{p}=5$ and $U / t_{p}=40$. Different curves correspond to different values of $t_{c} / t_{p}$ (upper and lower panels, respectively).

\section{Small $U: H M$ to $3 C O$ transition}

At low $U$, the instability toward the threefold charge ordered phase is signaled by a sharp jump in the correlation function [Fig. 3(a)], starting from a small constant value in the homogeneous metal. The locus of the 3CO transition shows an appreciable dependence on geometrical frustration: The homogeneous metal is rapidly destabilized for positive values of $t_{c} / t_{p}$, in marked contrast with the weaker (and opposite) variations expected from an RPA analysis valid in 

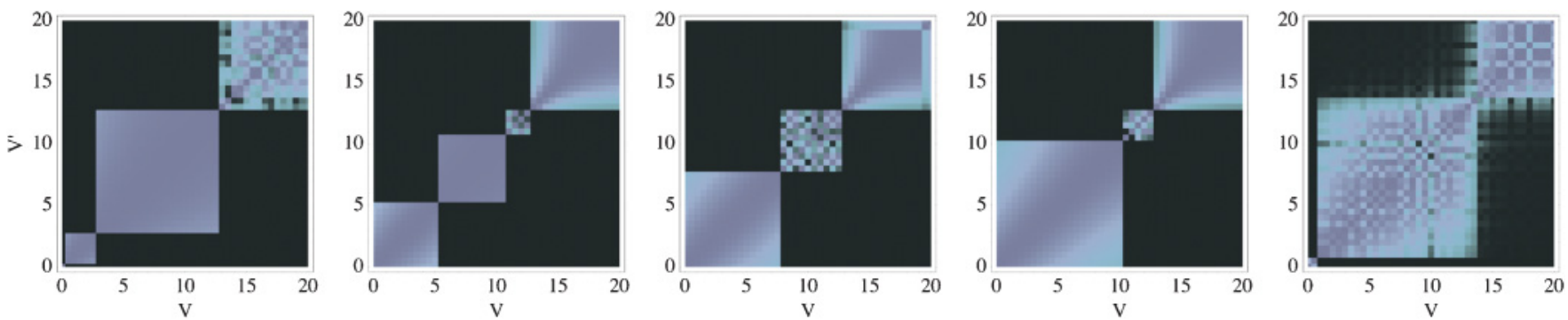

FIG. 5. (Color online) Fidelity $F_{V, V^{\prime}}=\left|\left\langle\psi_{0}\left(V^{\prime}\right) \mid \psi_{0}(V)\right\rangle\right|$ for various values of $t_{c} / t_{p}$, from left to right 1.0,0.5,0.0, -0.5 , and -1.0 and $U=40\left|t_{p}\right|$. There is a perfect agreement with the phase transitions obtained by standard quantities (order parameters, kinetic energies, susceptibilities). The case of zero overlap is represented in black, while the blue-violet (diagonal squares) represents overlap 1 ( $\left.V=V^{\prime}\right)$. The nonuniform-colored zones stand for fluctuating ground states possibly related to a strong degeneracy.

the weakly correlated limit $^{29}$ (see Appendix A). From the phase diagram of Fig. 2 it is quite clear that this trend is governed by a mechanism that extends from the strongly correlated limit $U \gg t_{p}$ down to the lowest values of $U$. The emergence of an intermediate plateau in the charge correlation function, clearly visible in the data at $t_{c}=t_{p}$ in Fig. 3(a), is also reminiscent of the situation encountered at $U / t_{p}=40$ (see below). These observations suggest that the presence of geometrical frustration, $t_{c} / t_{p}>0$, strongly enhances the role of electronic correlations. The pinball phase characteristic of strong $U$ is stabilized at $t_{c}=t_{p}$ despite a relatively low nominal value $U / t_{p}=5$.

We note that the nature of the ordering transition changes in the opposite limiting case $t_{c}=-t_{p}$, where the sharp jump in the correlation function is replaced by a smoother evolution, possibly due to the competition with an incipient nesting instability (Appendix A).

The behavior of the charge correlation function is directly mirrored in the other physical quantities shown in Figs. 3. The kinetic energy [Fig. 3(c)] jumps at the phase transition from an essentially free-electron value, $K / K_{0} \gtrsim 0.9$, to a value that is reduced by the opening of the charge ordering gap. At the same time, the double occupancy [Fig. 3(e)] undergoes a marked increase toward the value $d=0.66$ of the fully formed 3CO: The charge is ordered into three sublattices with average occupations $n_{A}=n_{B}=2$ and $n_{C}=1 / 2$ (see Fig. 4, large $V$ region), so that each of the two charge-rich sublattices contributes $d_{A}=d_{B}=1 / 3$ to the average double occupancy. The fact that the double occupancy in the homogeneous metal is suppressed from the noninteracting value $d=(n / 2)^{2}=$ $(3 / 4)^{2}=0.5625$ indicates the presence of moderate electronic correlations.

\section{Large $U$ : HM to PL transition}

A richer situation is found in the large- $U$ regime. First of all, the homogeneous liquid is characterized by a total suppression of double occupancy: By introducing the double occupancy of holes $d_{h}=\left\langle\left(1-n_{i \uparrow}\right)\left(1-n_{i \downarrow}\right)\right\rangle=1-n+d$ and setting $d_{h}=0$ we obtain $d=n-1=1 / 2$, which is actually observed in Fig. 3(f). Furthermore, the presence of both local and nonlocal Coulomb interactions hinders the particle motion, resulting in a marked reduction of the kinetic energy upon increasing $V$. An approximate expression for its $V$ dependence is

$$
K^{(\mathrm{HM})} \approx\left(1-A V^{2}\right) K_{U}^{(\mathrm{HM})},
$$

where $K_{U}^{(\mathrm{HM})}$ is the value at $V=0$. This $V^{2}$ dependence is consistent with a previous slave-boson ${ }^{48}$ calculation of the metallic phase formed by spinless particles on a $d$-dimensional hypercubic lattice, which is compatible with the data of Fig. 3(d). Because the ordering instability is pushed to large values of $V$ for negative values of $t_{c} / t_{p}$ (cf. Fig. 2), the homogeneous metal that is so revealed can acquire quite a strongly correlated character, as testified by a kinetic energy ratio that decreases down to $K / K_{0} \simeq 0.5$ before the onset of charge order.

The numerical data of Figs. 3(b) and 3(d) show quite clearly that an intermediate phase emerges between the homogeneous metal and the 3CO, which we associate with the pinball-liquid phase introduced by Hotta and coworkers. ${ }^{34,35,50}$ The PL is a partially ordered phase with a three-sublattice structure, in which the carriers of one sublattice (pins) are localized as a Wigner crystal and the remainder (balls) form an essentially noninteracting liquid within the resulting hexagonal lattice [Fig. 1(b)].

To get further insight about the different broken-symmetry phases, we have calculated the static density profile in the presence of a local perturbation breaking all the translations of the lattice, but respecting the $\pi / 3$ rotation. In this way, instead of obtaining a uniform linear combination of all symmetry-related crystal states, the system selects one crystal state favored by the perturbation, giving access to a real-space snapshot of the broken-symmetry ground state. The basis of the method employed for getting the real-space snapshot of the local densities is the following: We add a local potential on certain sites related by rotations in such a way that only translations are broken. The value of the local defects is a few percent of the hopping term. In the Hamiltonian, we simply add the perturbation term: $\delta \sum_{i}\left(n_{i \uparrow}+n_{i \downarrow}\right)$. This method has previously been used for distinguishing the exact nature of two phases breaking the translational and the rotational symmetries differently. ${ }^{51}$ Since the additional term is kept very small, it only corresponds to a perturbation and does not change the main property of the ground state. In this paper, we do want to keep the rotational symmetry safe; we hence chose 4 sites, in the 12-site cluster, in such a way that the system is still 
rotationally invariant. The perturbation has to be lower than the typical energy scale and we put $\delta=+0.05 t_{p}$ on the sites of the hole-rich sublattice. Results are depicted in Fig. 4. Expressing the charge densities in terms of holes, $n_{h}=2-n=1 / 2$, and starting from the $3 \mathrm{CO}$ phase $\left(n_{h, A}=n_{h, B}=0\right.$ and $n_{h, C}=3 / 2$, Fig. 4), by progressively increasing the local repulsion $U$, it becomes energetically unfavorable to accommodate more than one hole per molecule. Part of the hole density will then tend to spill out of the hole-rich sites in order to prevent double occupancy, resulting in $n_{h, C}=1$. The average charge density in the three sublattices, $n_{A}=n_{B}=7 / 4, n_{C}=1$, illustrated in Fig. 4 indeed corresponds to a scenario where one sublattice is occupied by localized holes $\left(n_{h, C}=1\right.$ leading to $n_{C}=$ $\left.2-n_{h, C}=1\right)$ with the remaining particles equally spread in the interstitial sites $\left(n_{h, A}=n_{h, B}=n_{\text {ball }}=1 / 4\right.$ hole per site, leading to $\left.n_{A}=n_{B}=2-n_{\text {ball }}=7 / 4\right)$. The critical value for the transition from $3 \mathrm{CO}$ to PL can be readily estimated to be $U \simeq 3 V$ from electrostatic considerations alone (see Appendix B), and is therefore independent of $t_{c} / t_{p}$.

Notably, in the PL phase the physical quantities whose $V$ dependence is depicted in Fig. 3 form well-defined plateaus, suggesting that they are locked as a consequence of the spontaneous separation between localized and itinerant charges. For example, the charge correlation function tends to the value $C(\mathbf{Q})=n^{2} / 3 \simeq 0.08$ instead of the full $C(\mathbf{Q})=n^{2}$ obtained at complete ordering in the large- $V$ limit, corresponding to the fact that only $1 / 3$ of the particles participate to the ordering phenomenon.

From our numerical results in Fig. 3(d), the kinetic energy of both the homogeneous metal at $V<V_{\mathrm{PL}}$ and the $3 \mathrm{CO}$ phase at $V>V_{3 \mathrm{CO}}$ is found to depend only weakly on the degree of geometrical frustration $t_{c} / t_{p}$. On the other hand, the kinetic energy forms a plateau within the PL phase, at a value which is strongly dependent on $t_{c} / t_{p}$ : The absolute value of the (negative) kinetic energy in the PL phase is lowest at $t_{c}=-0.5 t_{p}$ and its magnitude steadily increases as the frustration ratio is increased to $t_{c}=t_{p}$. Our data therefore suggest that it is this gain in kinetic energy for positive values of the geometrical frustration ratio that is responsible for the stabilization of the pinball-liquid phase against the homogeneous metal observed in Fig. $2 .^{49} \mathrm{We}$ note that a kinetic-energy-driven mechanism for the PL transition was also pointed out in Ref. 35. Since the effective filling associated with the itinerant balls on the hexagonal lattice is only $n_{\text {ball }}=1 / 8$, Coulomb interaction effects are small. Indeed, we have checked that the kinetic energy of the balls coincides with the kinetic energy of the corresponding noninteracting tight-binding model on a hexagonal lattice, at filling $n=n_{\text {ball }}$, as a function of the ratio $t_{c} / t_{p}$.

\section{B. Fidelity analysis}

In order to characterize the quantum phase transitions of our system, the simple yet powerful concept of fidelity ${ }^{52}$ is considered here (Fig. 5). Initially introduced in quantum information, the fidelity has proven to be successful in determining superfluid-insulator transitions of the Hubbard model. ${ }^{53-55}$ The idea behind the fidelity is very simple; it consists of computing overlaps of ground states (GS) $\left|\psi_{0}\right\rangle$ at different values of the microscopic parameters. At a QCP, even the smallest change of the parameters can have dramatic effects in some of the observables. This is encoded in the GS properties; hence, the overlap is expected to strongly react and indicate the locations of the phase transitions. We define the fidelity $F$ as

$$
F_{V, V^{\prime}}=\left|\left\langle\psi_{0}\left(V^{\prime}\right) \mid \psi_{0}(V)\right\rangle\right| .
$$

Obviously, for $V=V^{\prime}$, the fidelity should be $F_{V, V}=1$. Typical results are depicted in Fig. 5 at $U=40 t_{p}$, and for five values of $t_{c} / t_{p}= \pm 1.0, \pm 0.5$, and 0.0 .

It is surprising to see how the fidelity is indeed able to pinpoint the phase transitions. For each of the values of $t_{c} / t_{p}$, we exactly recover the transitions obtained by more standard methods in the preceding sections. More information is available, however. First, there is always some region where $F$ is strongly fluctuating, even though well delimited in the $\left(V, V^{\prime}\right)$ plane (nonuniform-colored zones). These fluctuations can be due to a large degeneracy of the GS hence corresponding to a same order but with destructive interferences. The second important piece of information is visible in the case $t_{c} / t_{p}=0.5$, where the fidelity indicates the existence of two distinct phases in the pinball region [namely $5.20(2) \leqslant V / t_{p} \leqslant 11.00(2)$ and $\left.11.00(2) \leqslant V / t_{p} \leqslant 13.00(2)\right]$, suggesting a possible ordering of the mobile charges. In fact, a slight change in the kinetic energy [Fig. 3(d)] appears at this transition, but the other quantities seem to be insensitive to it. The fact that the transition within the pinball phase is not detected by the charge correlation function [Fig. 3(b)] nor by the average densities (Fig. 4) tends to show that their properties remain extremely close. Nevertheless, the fidelity $F$ allows a precise determination of the subphases.

\section{Spinless model}

We now consider the spinless version of the model Eq. (1), which has been discussed extensively in the literature. ${ }^{34,35,50,56}$ Since the spinless model only contains charge degrees of freedom, by comparing it with the spinful model we can obtain useful information on the relative role played by charge fluctuations as compared to the spin fluctuations.

The spinless model reads

$$
\begin{aligned}
H= & t_{p} \sum_{\langle i j\rangle_{p}}\left(h_{i}^{\dagger} h_{j}+\text { H.c. }\right)+t_{c} \sum_{\langle i j\rangle_{c}}\left(h_{i}^{\dagger} h_{j}+\text { H.c. }\right) \\
& +V_{p} \sum_{\langle i j\rangle_{p}} n_{i} n_{j}+V_{c} \sum_{\langle i j\rangle_{c}} n_{i} n_{j},
\end{aligned}
$$

where we have changed the sign of the hopping integrals to deal explicitly with holes. As in the preceding sections we consider the case $V_{c}=V_{p}=V$ for different values of the $t_{c} / t_{p}$ ratio. Importantly, for spinless particles the physical situation of one hole per two sites implies a half-filled band, which gives rise to a spurious particle-hole invariance that is absent in the spinful case at 3/4-filling. Therefore, the thermodynamic properties as well as the phase transition lines become invariant under a change of sign of $t_{c} / t_{p}$.

In Fig. 6 we show the $V$ dependence of the kinetic energy for different $t_{c} / t_{p}$ ratios compared with the spinful model. As expected the figure shows how the kinetic energy in the spinless model does not depend on the sign of $t_{c} / t_{p}$ (black 


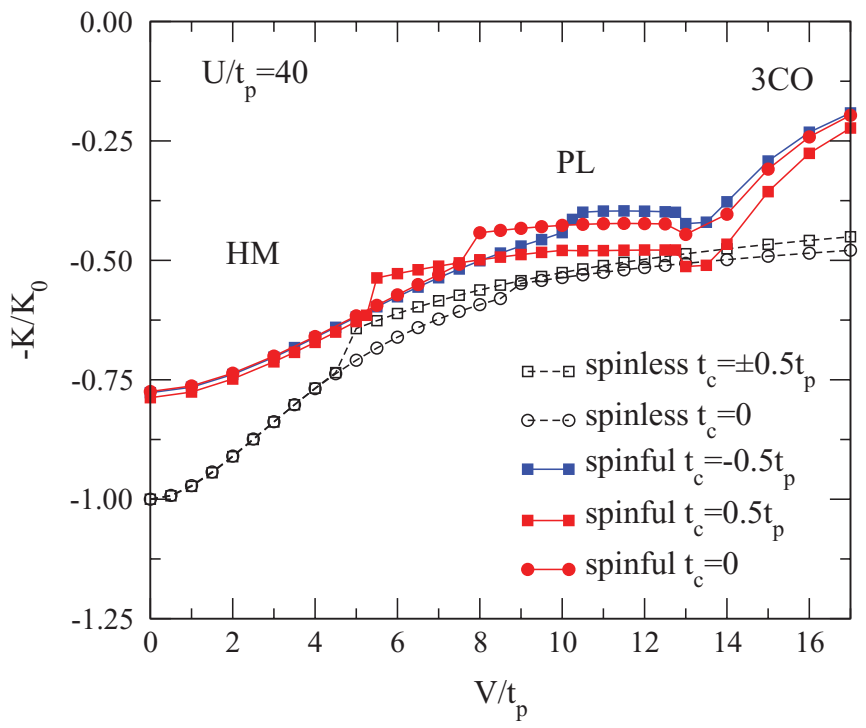

FIG. 6. (Color online) Dependence of the kinetic energy on $V$ for the spinless and spinful extended Hubbard models. Different $t_{c} / t_{p}$ ratios are compared in the two situations. As expected, the properties of the spinless model are independent of the sign of $t_{c} / t_{p}$, in contrast to the spinful model.

curves). The spinless model is able to recover the qualitative trends of the full model for $t_{c} / t_{p} \geqslant 0$ : The critical values at which the transition from the HM to the PL occurs are respectively $V_{\mathrm{PL}} \simeq 4.5 t_{p}$ and $8.5 t_{p}$ for $t_{c} / t_{p}=0.5$ and 0 in the spinless case, to be compared with $V_{\mathrm{PL}} \simeq 5.5 t_{p}$ and $7.5 t_{p}$ in the spinful case at $U=40 t_{p}$ (Fig. 6). However, because of the artificial particle-hole symmetry, for $t_{c} / t_{p}<0$ the locus of the transition is completely inconsistent with the spinful case, and the stability of the PL is widely overestimated.

We see from Fig. 6 that the effect of nonlocal interactions $V$ on the renormalization of the kinetic energy in the homogeneous metal is very similar in the spinful model at large $U$ and in the spinless model, both being compatible with the quadratic $V$ dependence discussed above. We therefore conclude that the different behaviors observed in the two models at $t_{c} / t_{p}>0$ are a direct consequence of the different kinetic energies at the noninteracting level, which results from the spurious particle-hole symmetry acquired by the spinless version.

The results presented here indicate that the charge rather than spin correlations dominate the renormalization effects on metallic properties approaching the charge-order transition. However the spin multiplicity enters (indirectly) via the geometrical frustration, which is not treated correctly in the spinless model. A realistic spinful calculation therefore appears to be necessary to properly address the physics of $\theta$-(ET) $)_{2} \mathrm{X}$ salts, where $t_{c} / t_{p}$ is a key parameter in determining the experimental phase diagram. ${ }^{20,38}$

\section{CORRELATED METAL AT FINITE TEMPERATURES}

Here we analyze the properties of the homogeneousmetallic phase at finite temperatures close to the QCP. A temperature scale emerges, which we denote $T^{*}$, above which the kinetic energy departs from Fermi-liquid behavior and the specific-heat coefficient goes through a maximum. We interpret $T^{*}$ as a renormalized Fermi temperature, which generally drops to zero at the approach of the QCP. Such behavior is typically found for geometrical frustration $t_{c} \neq$ $\pm t_{p}$. On the contrary, in cases in which there are competing Fermi surface instabilities, especially in the perfectly nested case $t_{c}=-t_{p}$, the $T^{*}$ phenomenon is much weaker, and hardly affects the properties of the electron liquid. The data in that case are compatible with a Fermi temperature that remains finite right close to the QCP, possibly indicating that a first-order transition may be occurring.

In the following paragraphs we focus specifically on the realistic value $U=15 t_{p}$, but the qualitative features presented here are unchanged for different large values of $U$.

\section{A. Non-Fermi-liquid behavior close to $\mathrm{CO}$}

\section{Kinetic energy}

The temperature dependence of the average kinetic energy $K$ normalized to the noninteracting value $K_{0}(U=V=0)$ is shown in Fig. 7 for $t_{c} / t_{p}=1,0.5,0,-0.5,-1$. The different curves in each panel correspond to different values of the intersite Coulomb repulsion, $V$, across the charge-ordering transitions. The ratios $t_{c} / t_{p}=0.5,0,-0.5$ [Figs. 7(b), 7(c), and $7(\mathrm{~d})$ ] all show marked departures from the quadratic temperature dependence $K=K_{T=0}-B T^{2}$ characteristic of conventional metals, occurring in the HM phase above a certain temperature (the approximate locus of the inflection points is indicated by arrows). We denote it as $T^{*}$ and take it as an estimate of the renormalized Fermi temperature, governing a crossover to non-Fermi-liquid behavior. Clearly, $T^{*}$ is progressively reduced upon approaching the charge-ordering transition and vanishes at the critical point. In this respect, our data in the experimentally relevant case $U=15 t_{p}$ do not show qualitative differences between the transition to the threefold charge order obtained for $t_{c} / t_{p}=-0.5,0$ and that to the pinball phase for $t_{c} / t_{p}=0.5$ (cf. Fig. 2): In both cases the temperature scale $T^{*}$ appears to be entirely controlled by the approach to the zero-temperature ordering transition, indicating the possibility of quantum critical behavior at finite temperatures around the zero-temperature phase transition.

In the cases in which $t_{c}= \pm t_{p}$, the emergence of a temperature scale $T^{*}$ is much less clear [Figs. 7(a) and $7(\mathrm{e})]$ as the temperature dependence of the kinetic energy is smooth within the whole homogeneous-metallic phase except very close to the transition. Our RPA analysis presented in Appendix A shows that in these cases there is a competing charge-density wave (CDW) instability, which could indeed be masking the quantum critical behavior associated with the $3 \mathrm{CO}$ transition. For $t_{c} / t_{p}=-1$ the CDW is driven by the perfect nesting of the Fermi surface whereas for $t_{c} / t_{p}=1$ a mixed CDW/CO phase induced by both nesting tendencies and strong Coulomb repulsion exists.

The perfect nesting of the Fermi surface for $t_{c} / t_{p}=$ -1 occurs at the wave vectors $\mathbf{Q}_{F}=( \pm \pi, \pm \pi / \sqrt{3})$ and $(0, \pm 2 \pi / \sqrt{3})$. Such nesting instability is dominant at weak $U$, 

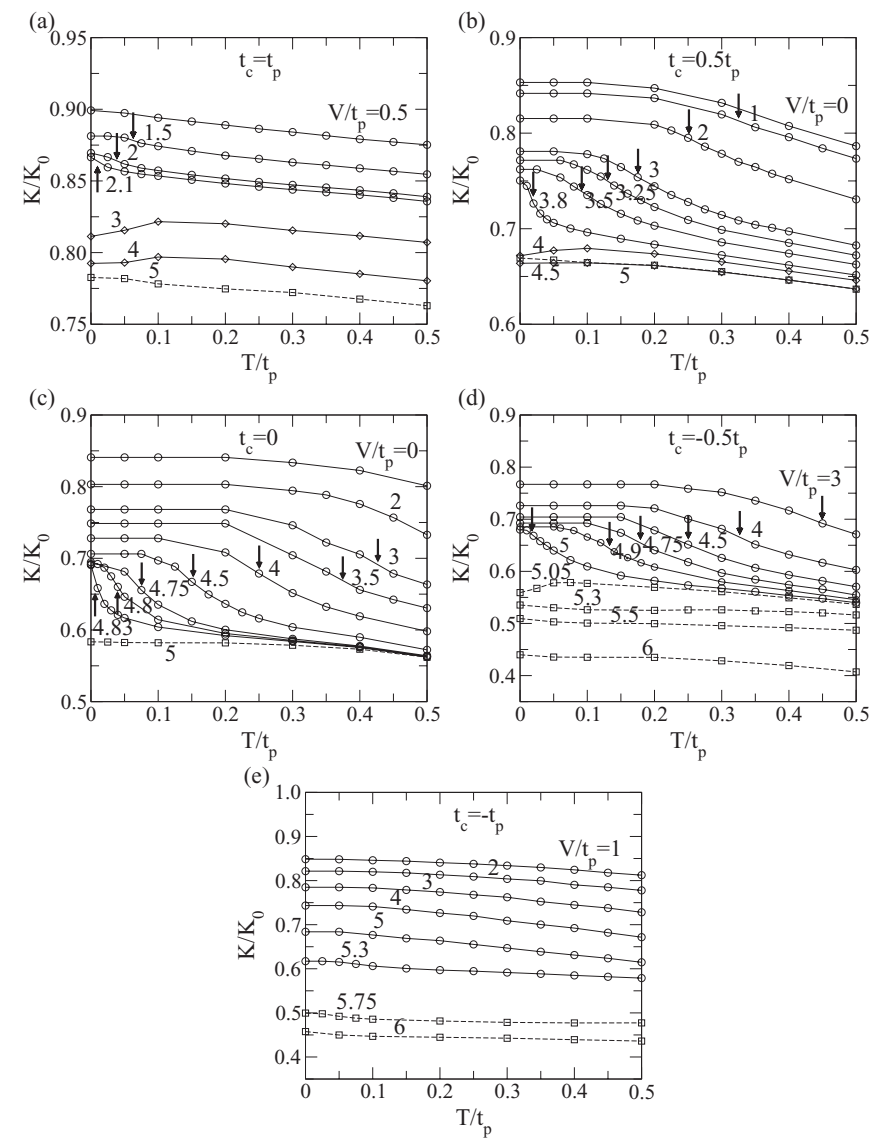

FIG. 7. Temperature dependence of the total kinetic energy at $U=15 t_{p}$ normalized to the noninteracting value $K_{0}$, for $t_{c} / t_{p}=$ $-1,-0.5,0,0.5$, and 1 . Arrows correspond to inflection points of the curves, indicating the temperature scale $T^{*}$ corresponding to the breakdown of the quasiparticles. Open circles correspond to the HM phase, diamonds to the PL (full lines), and squares (dashed lines) to the $3 \mathrm{CO}$ phase.

where it results in a striped charge modulation that dominates over the threefold charge order discussed above (this striped order is analogous to the checkerboard pattern obtained in the square lattice). Our data suggest that even in the presence of a sizable local Coulomb repulsion, $U=15 t_{p}$, that prevents the stabilization of such stripe order, an incipient nesting instability is strong enough to destroy the quantum criticality around the QCP. This conclusion is based on the observation that there is no clear signature of the vanishing low temperature scale $T^{*}$ at the QCP and there is no clear evidence of the "bad" metallic behavior found for other $t_{c} / t_{p}$ ratios. However, there is a weak $T$ dependence of the kinetic energy that vanishes at a critical value $V_{c}=6 t_{p}$ as can be observed from the data of Fig. 7(e). Such critical value, $V_{c}$, is found to be consistent with the critical value obtained from the $3 \mathrm{CO}$ charge correlations calculated below.

\section{Charge correlations}

The emerging QCP scenario can be further appreciated by studying the evolution of the charge-ordering transition $T_{\mathrm{CO}}$ vs temperature. This can be obtained by tracking the steepest variation of the charge correlation function, $C(\mathbf{Q})$ (a)

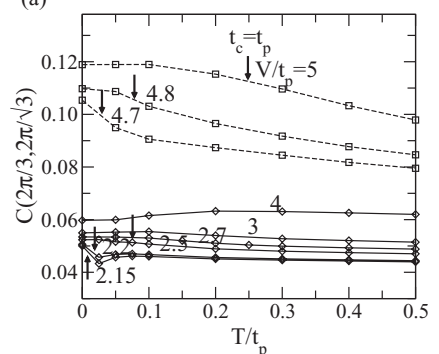

(b)
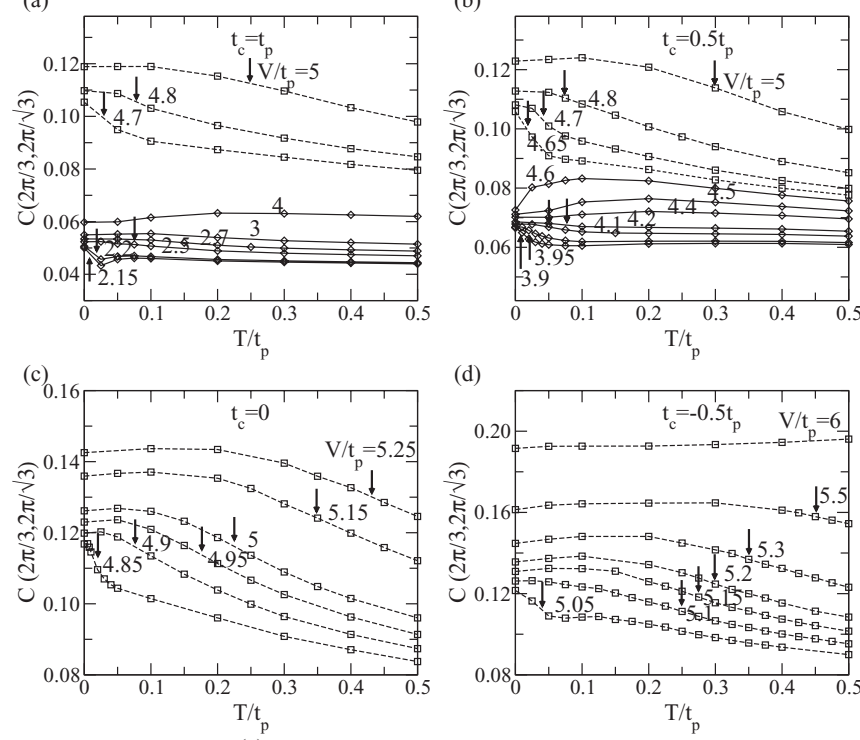

(d)

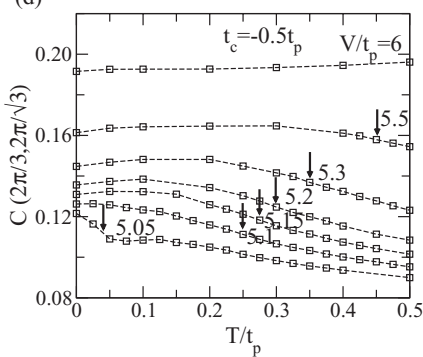

(e)

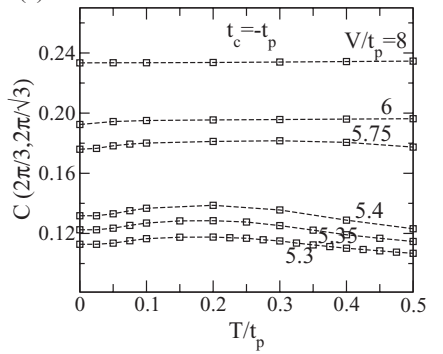

FIG. 8. Charge correlation function versus the temperature for different $V / t$ in the $\mathrm{CO}$ phase at $U=15 t$ for $t_{c} / t_{p}=1,0.5,0$, $-0.5,-1$. Arrows correspond to inflection points of the curves, indicating the ordering temperature $T_{\mathrm{CO}}$.

[arrows in Figs. 8(a)-8(d)]. In Fig. 9 we report $T_{\text {CO }}$ together with the $T^{*}$ extracted from Fig. 7, showing a common behavior in proximity to the $\mathrm{CO}$ instability. As stated in the preceding paragraph, the case $t_{c} / t_{p}=-1$ exhibits a different behavior, with no visible $T^{*}$ approaching the QCP. The charge correlations [Fig. 8(e)] also exhibit a qualitatively different behavior in the perfectly nested case $t_{c} / t_{p}=-1$, with a mild nonmonotonic temperature dependence showing a maximum at intermediate temperatures which suggests a "reentrant" ordering transition. For this case, there is no clear indication of "bad" metallic behavior in the kinetic energy and there is clear evidence of "reentrant" behavior in the 3CO transition. This reentrant behavior disappears at around the critical value $V_{c}=6 t_{p}$ as can be noted in Fig. 8(d). It can be noted that a slightly reentrant behavior can be extracted from the data at $t_{c} / t_{p}=-0.5$. The presence of a reentrant behavior at negative values of $t_{c} / t_{p}$ is confirmed by our RPA analysis, and is strongly reminiscent of what is commonly observed in the EHM on the square lattice (cf. Fig. 1 in Ref. 14), which also in that case is ascribed to the competition of the $\mathrm{CO}$ phase with a Fermi surface nesting instability. We have added to the phase diagram for the case $t_{c}=-t_{p}$ the $2 k_{F}-\mathrm{CDW}$ instability and may also be present in other cases. However, the limited wave vector resolution of our small-cluster calculation does not permit an accurate determination of the stability of the $2 k_{F}$-CDW phase. In fact, for $t_{c}=-0.5 t_{p}$, we may also expect 

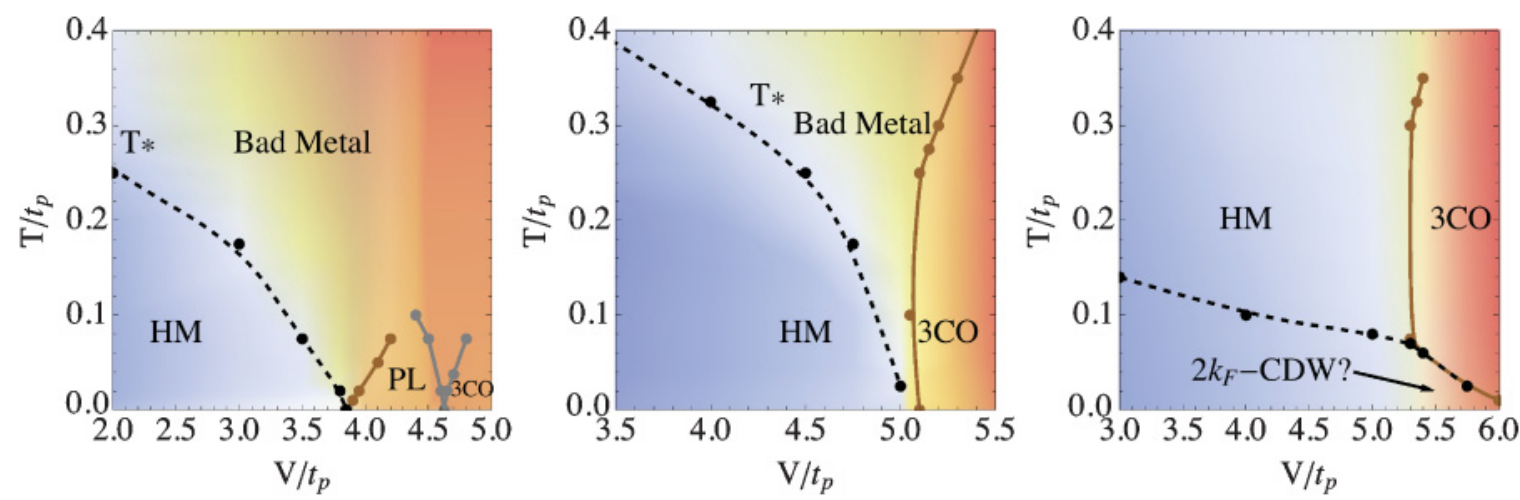

FIG. 9. (Color online) Color plots summarizing the QCP behavior at $U=15 t_{p}$ for the representative cases $t_{c} / t_{p}=0.5,-0.5$, and -1 . The dashed curve is the renormalized Fermi temperature $T^{*}$ extracted from Fig. 7. The full line is the charge ordering temperature $T_{\mathrm{CO}}$ extracted from Fig. 8. The color gradients (blue and red, respectively) are derived from the kinetic energy and charge correlation data of Figs. 7 and 8.

that $\mathrm{CDW}$ instabilities occur in the proximity of the $3 \mathrm{CO}$ instability.

\section{Specific heat}

Further insight on the anomalous properties of the homogeneous phase can be gained by exploring thermodynamic properties such as the temperature dependence of the specificheat coefficient $C_{V} / T$ on approaching the QCP. In a Fermi liquid at low $T$, this quantity measures the effective mass enhancement of the quasiparticles. In Fig. 10 we compare $C_{V} / T$ for $t_{c} / t_{p}=0.5,-0.5,-1$. In this way we compare the behavior of the specific heat of a system across the 3CO $\left(t_{c}=-0.5 t_{p}\right)$ with a system across the PL transition $\left(t_{c} / t_{p}=\right.$ $0.5)$. For completeness we also analyze the $t_{c} / t_{p}=-1$ case in which perfect nesting exists which can be compared to the other two cases. For both $t_{c} / t_{p}= \pm 0.5$ we find that a peak in $C_{V} / T$ develops at $T^{*}$ with $T^{*} \rightarrow 0$ on approaching the QCP as $V \rightarrow V_{c}$. This indicates that both the drop of $T^{*}$ and the effective mass enhancement occurring in proximity to the QCP are consistent with the phase diagram of Fig. 9. Both effects are key signatures of the presence of a QCP together with the bad metallic behavior arising around it.

The case $t_{c}=-t_{p}$ deserves special attention. For Coulomb repulsion energies up to about $V=5.4 t_{p}$, there is a moderate increase of the effective mass enhancements and a moderate shift of the peak to lower temperatures in contrast to previous cases. However, increasing $V$ further leads to different behavior with a rapid increase of the effective mass enhancement and a shift of the peak to zero which indicates the proximity to a $3 \mathrm{CO}$ transition at $V_{c}=6 t_{p}$. The shift of the peak in the specific heat with $V$ is plotted in the phase diagram of Fig. 9 together with the transition line to the $3 \mathrm{CO}$. The two lines merge and a clear reentrant behavior of the $3 \mathrm{CO}$ transition is observed which is ascribed to the presence of the competing Fermi surface nesting instability. The origin of the specific-heat peak is unclear since it does not separate the HM from the bad metal and from Fig. 9 it is clear that the peak position has a different qualitative behavior for $t_{c} / t_{p}=-1$ than for other $t_{c} / t_{p}$ ratios. In fact, there is no bad metallic behavior in the HM phase as discussed previously. However, the fact that the effective mass is enhanced may indicate a transition to the $2 k_{f}$-CDW.

\section{B. Connection with QCP physics}

It is worth analyzing our numerical results from the perspective of the standard Moriya-Hertz-Millis (MHM) $)^{57,58}$ theory of quantum critical points (QCPs). In principle, this theory could be appropriate to the transition from the HM to the $3 \mathrm{CO}$ phases considered here since both are metallic.
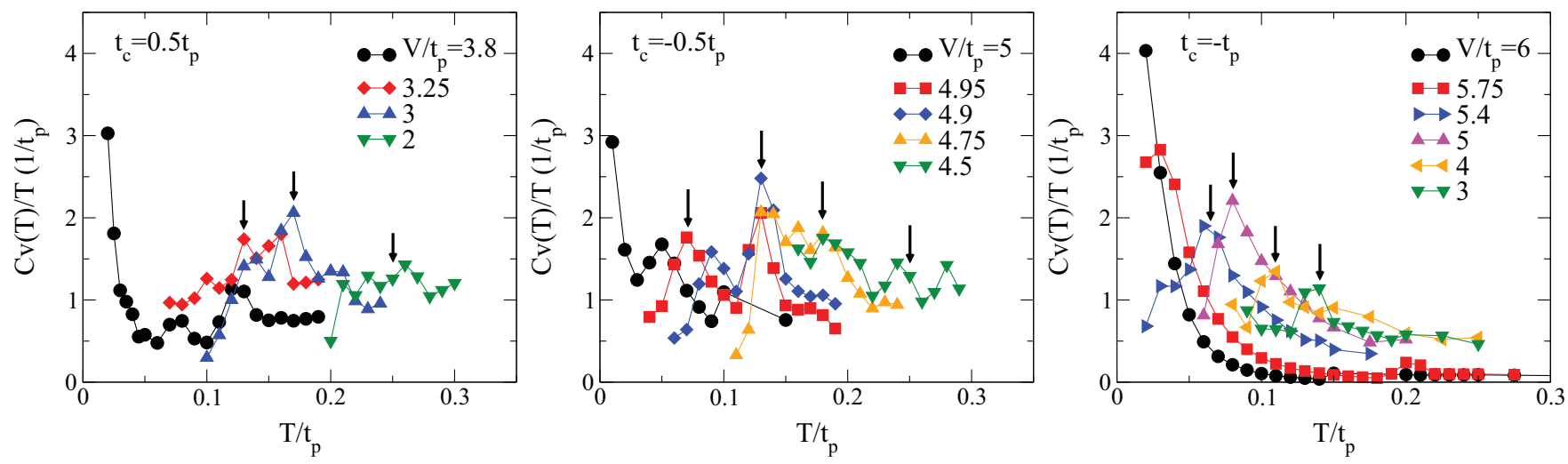

FIG. 10. (Color online) Temperature dependence of effective mass enhancement for different $t_{c} / t_{p}$ ratios for $U=15 t_{p}$. A different behavior in the $T$ dependence is found for the case $t_{c}=-t_{p}$ for $T^{*}$ (indicated by the vertical arrows) which indicates a departure from the quantum critical behavior found in the other non-nested Fermi surface situations. 
However, one should keep in mind the limitations of the MHM theory. First, it is based on a weak-coupling perturbative expansion around the QCP. Second, the MHM theory is not applicable for ordering transitions which are driven by " $2 \mathrm{k}_{f}$ " Fermi surface instabilities. Hence, the MHM theory is applicable only to systems in which there is no nesting at all and for which the ordering wave vector $\mathbf{Q}$ is not an extremal vector of the Fermi surface, i.e., loosely speaking, $Q \neq 2 k_{f}$. Our model satisfies the latter condition whenever $t_{c} / t_{p} \neq \pm 1$ for the $3 \mathrm{CO}$ wave vector: $\mathbf{Q}=(2 \pi / 3,2 \pi / \sqrt{3})$ (see Fermi surfaces and discussion in Appendix A). In these cases, the two-dimensional version of MHM, $d=2$, with a dynamical scaling dimension, $z=2$ (corresponding to nearly antiferromagnetic metals with effective dimension at the QCP: $D=d+z=4)$, is relevant. This situation corresponds to a marginal case which contains dangerously irrelevant operators in the renormalization group sense which can destroy the hyperscaling at the QCP. In this marginal case, $d=z=2$, the Fermi-liquid phase is bounded in the phase diagram by the condition $T<r$, where $r=\left|V-V_{c}\right|$ quantifies the proximity to the QCP from the metallic side of the transition and $V_{c}$ is the critical value at which $\mathrm{CO}$ occurs. The quantum critical region is bounded by the condition $T>r$ obtained from the scaling behavior of the renormalization group (RG) equations. A similar linear dependence with $r$ is found in the boundary of the ordered phase which displays a critical nonGaussian behavior around it due to the Coulomb interaction. As summarized in Fig. 9, for $t_{c} / t_{p} \neq \pm 1$ the suppression of $T^{*}$ at the QCP and the bad metallic behavior obtained at finite $T$ from our numerical calculations are qualitatively consistent with the MHM predictions for the marginal $d=z=2$ case.

On the other hand, the extremal values, $t_{c} / t_{p}= \pm 1$, deserve special consideration. In the particular case $t_{c}=-t_{p}$, the system has perfect nesting at the ordering wave vector, $\mathbf{Q}_{F}=(\pi, \pi / \sqrt{3})$ (see Fig. 11), which describes diagonal stripe order in real space. A RPA analysis on the model shows that a CDW instability at $\mathbf{Q}_{F}$ exists at small but finite $U$ and $V$, which competes with the $3 \mathrm{CO}$ with $\mathbf{Q}=(2 \pi / 3,2 \pi / \sqrt{3})$. Such coexistence/competition between $\mathrm{CDW}$ and $\mathrm{CO}$ instabilities has also been found in RPA studies of the extended Hubbard model on the square lattice. ${ }^{14}$ The situation in which two instabilities coexist - a nesting-driven CDW with ordering vector $\mathbf{Q}_{F}$ and a Coulomb-driven instability with ordering vector $\mathbf{Q}$ - has not been addressed in general at the level of the MHM approach. Our analysis shows that at moderate values of $U \gtrsim 7 t_{p}$ nesting instabilities are washed away and the charge-ordering transition is Coulomb driven. However, the competition washes out the $T^{*}$ phenomenon and we find no clear evidence of bad metallic behavior around the QCP in the case $t_{c}=-t_{p}$. This coincides with the breakdown of the MHM approach when nesting is present in the lattice.

We may speculate, based on our numerical analysis, that in the perfectly nested situations quantum criticality is destroyed and a first-order transition occurs. Indeed, a somewhat related renormalization group (RG) approach ${ }^{59}$ to $2 k_{F}$-density-wave quantum phase transitions in which curved Fermi surfaces with parallel tangents at two points of the Fermi surface connected by $2 k_{F}$ are considered has found that critical fluctuations strongly influence the fermions on the Fermi surface and that the feedback effect of these fluctuations can destroy the second-order quantum critical point turning it into a first-order transition. Only in the special case in which $\mathbf{Q}=\mathbf{G} / 2$, with $\mathbf{G}$ being a reciprocal lattice vector, a second-order quantum phase transition is recovered.

In actual quarter-filled organic materials, $\theta-\mathrm{ET}_{2} \mathrm{X}$, for which hopping ratios $t_{c} / t_{p} \neq-1$, the MHM theory may be relevant. Many of the predictions for thermodynamic and transport properties in the quantum critical regime above the zero-temperature QCP could be then experimentally checked. One important prediction of the MHM theory for $d=z=2$ is the anomalous temperature dependence of the specific heat:

$$
\begin{gathered}
\left.C \sim T \frac{\partial S}{\partial T}\right|_{V} \sim T \ln 1 / r, \quad T \ll r, \\
T \ln 1 / T, \quad T \gg r,
\end{gathered}
$$

where $r$ describes the proximity to the QCP. On the other hand, in clean nearly charge ordered two-dimensional metals the resistivity around the "hot" spots in the quantum critical regime $^{19,60}$ reads

$$
\begin{gathered}
\rho \propto T^{2}, \quad T \ll r, \\
T, \quad T \gg r .
\end{gathered}
$$

However, the resistivity is short-circuited by the contribution of electrons at the "cold" spots since the scattering is small around these parts. ${ }^{61}$ Hence, the non-Fermi-liquid behavior at the hot spots is masked by the cold sections eventually restoring Fermi-liquid behavior: $\rho \sim T^{2}$. Therefore, within the MHM approach and in the the quantum critical region, the specific-heat coefficient displays divergent behavior as $T \rightarrow 0$ following Eq. (10). The resistivity could show non-Fermiliquid behavior under small disorder which has been found to strongly influence antiferromagnetic QCPs. ${ }^{62}$ Averaging the scattering rate over the Fermi surface reduces the effectiveness of the Hlubina-Rice mechanism and the scattering from the hot regions becomes effective leading again to non-Fermi-liquid behavior. $^{62}$

The behavior of the specific-heat coefficient that we have found around the critical point (see Fig. 10) does show an enhancement on approaching the QCP in consistent agreement with Eq. (10). However, we cannot accurately determine whether a logarithmic dependence occurs in our numerical darta due to the small cluster sizes reached.

\section{CONCLUSIONS AND OUTLOOK}

We have analyzed in detail the effect of geometrical frustration on charge-ordering transitions realized in the extended Hubbard model on the anisotropic triangular lattice, which appropriately describes the family of quarter-filled layered organic crystals: $\theta-(\mathrm{ET})_{2} \mathrm{X}$. The model contains both on-site, $U$, and intersite Coulomb repulsion terms $V_{p}$ and $V_{c}$, which are taken to be isotropic, $V_{c}=V_{p}=V$. The degree of geometrical frustration in the electron motion is tuned through the $t_{c} / t_{p}$ ratio, which is an important parameter controlling the experimental phase diagram.

The zero-temperature phase diagram of this model contains a homogeneous-metal (HM), a pinball-liquid (PL), and a threefold charge ordered $(3 \mathrm{CO})$ phase. While the $3 \mathrm{CO}$ phase occurs at sufficiently strong intersite interactions $V$ for any 
fixed $U$, the PL only occurs above a certain threshold $U$ value, as its existence is inherently tied up to the strong coupling regime. On the other hand, the PL is found to be stabilized by increasing the geometrical frustration of the lattice. Our results do show that in the range of values of $U / t_{p}$ and $V / t_{p}$ appropriate to the $\theta-(\mathrm{ET})_{2} \mathrm{X}$ materials, increasing the geometrical frustration of the lattice can effectively tune the system from a homogeneous metal (HM) with strong charge-order correlations to a pinball-liquid (PL) phase.

The phase transitions between charge-ordered and - disordered metallic phases can display quantum critical phenomena in close analogy with the heavy-fermion systems ${ }^{16-19}$ with the critical charge rather than the spin fluctuations driving the CO transition. Such type of fluctuations may be at the origin of both the anomalous properties in the metallic state and Cooper-pair formation. Indeed, non-Fermi-liquid behavior as well as non-BCS superconductivity have both been predicted and observed in quarter-filled organic materials of the $\alpha, \beta^{\prime \prime}$, and $\theta-(\mathrm{ET})_{2} \mathrm{X}$ type ${ }^{63-67}$ Such heavy-fermion behavior arising from molecular $\pi$ electrons instead of the $d$ or $f$ electrons, as occurs in the rare earths, may indeed find a natural explanation based on the properties of matter expected near a QCP.

In order to establish whether quantum critical behavior occurs or not in the quarter-filled layered materials close to $\mathrm{CO}$ several issues could be experimentally and theoretically addressed: (i) Is there evidence for the divergence of the specific-heat coefficient and the quasiparticle effective mass, $m^{*} / m \rightarrow \infty$, and for the collapse of the Fermi temperature, $T^{*} \rightarrow 0$, near the QCP? Measurements of the quadratic coefficient of the resistivity approaching the QCP from the Fermi-liquid side of the critical point can be useful to test the effective mass enhancement. Such type of experiments have been systematically performed in $\kappa$-(DHDA-TTP $)_{2} \mathrm{SbF}_{6}$ and $(\mathrm{MeDH}-\mathrm{TTP})_{2} \mathrm{AsF}_{6}$, by tuning the system across the $\mathrm{CO}$ transition via applied pressure ${ }^{64,65}$ yielding phase diagrams similar to those of Fig. 9. (ii) Is there non-Fermi-liquid behavior of transport and thermodynamic properties in the quantum critical regime above the QCP? What is the temperature dependence of the resistivity in these systems? Are there clear deviations from Fermi-liquid behavior of the form in Eqs. (10) and (12)? (iii) If quantum criticality and scaling are observed in transport and thermodynamic quantities, how much of this behavior is consistent with the MHM predictions? Could there be a new universality class around the QCP in quasi-two-dimensional organic materials, related to the emergence of the pinball phase? (iv) Measurements of the Hall coefficient can be useful to disentangle whether the QCP is of the MHM type or different. In standard MHM theories, the Fermi surface would fold due to Bragg reflection off the density wave with no discontinuity in the Hall constant when the system is tuned across the QCP. ${ }^{17,18}$ However, as in heavy fermions a local type of QCP could arise in which the system jumps discontinuously from a large Fermi surface to a small Fermi surface through the QCP leading to a discontinuous jump of the Hall coefficient. Since the transition from the HM to the PL involves localization of the "pin" electrons, a transition from the large Fermi surface of the HM involving all carriers to a small Fermi surface involving only "ball" itinerant electrons could indeed occur. Understanding how this transition takes place and the type of QCP observed could be resolved by Hall constant measurements in analogy to the heavy-fermion systems. (v) Here we have mainly discussed transitions between disordered and ordered metallic phases for which MHM theory is meant. An important issue to address is how quantum criticality is modified in transitions from HM to $\mathrm{CO}$ insulating phases. This issue can be addressed within the EHM studied in the present work, by allowing for anisotropic Coulomb interactions $V_{p} \neq V_{c}$.

As observed in Refs. 68 and 68 the superconductivity in $\theta$-(ET $)_{2} \mathrm{X}$ compounds, as in other polytypes, frequently appears near to an insulator. In such cases, the cause of superconductivity (SC) may not be the simple weak-coupling BCS mechanism by the electron-phonon interaction, but rather due to electronic correlations. Several theoretical works in the weak-coupling limit have been performed in order to examine the possible mechanism for the onset of SC in proximity to the $\mathrm{CO}$ phase. ${ }^{67,70,71}$ Unconventional SC of the $f$-wave type has been encountered on the anisotropic triangular lattice with the model parameters $t_{c}=0$ and $V_{c}=V_{p} \approx t_{p}{ }^{36}$ with $U=10 t_{p}$ and mediated by the charge fluctuations. This $f$-wave pairing symmetry is the analogous to the $d_{x y}$-wave pairing found in proximity to the checkerboard $\mathrm{CO}$ on the square lattice. ${ }^{67}$ It would be interesting to search for unconventional SC around the QCP found for other $t_{c} / t_{p} \neq 0$ ratios and $U$ values both in the $3 \mathrm{CO}$ and PL type QCP. Based on the results of the present work, it can be conjectured that the anomalous properties and unconventional superconductivity observed in $\mathrm{X}=\mathrm{I}_{3}$ compounds maybe related to the proximity to the strong-coupling PL phase since the on-site Coulomb repulsion energy is significant: $U=(15-20) t_{p}$. This could be tested by applying uniaxial pressure on the $\theta-(\mathrm{ET})_{2} \mathrm{I}_{3}$ crystals.

Other materials such as the rare-earth nickelates, $\mathrm{AgNiO}_{2}$, do show threefold $\mathrm{CO}$ transitions similar to the one discussed here $^{72}$ although the origin of the $\mathrm{CO}$ transition may be nonCoulomb in origin since $\mathrm{AgNiO}_{2}$ has a complex multiorbital structure $^{73}$ and other effects such as Hunds coupling and crystal fields can play a relevant role. Thus, the organic materials of the $\theta$-(ET) $)_{2} \mathrm{X}$ type appear to be ideal candidates to single out the effects on electronic properties of metals close to a charge-driven quantum phase transition mediated solely by the off-site Coulomb repulsion between electrons at different sites.

\section{ACKNOWLEDGMENTS}

We acknowledge A. Greco, P. Horsch, A. Liebsch, and M. Tamura for fruitful discussions. J.M. and L.C. acknowledge financial support from MICINN (CTQ-2008-06720-C02-02 and Consolider CSD2007-00010).

\section{APPENDIX A: RANDOM PHASE APPROXIMATION RESULTS AT WEAK $U$}

In the random phase approximation (RPA), the instability of the homogeneous metal is signaled by a divergence of the charge susceptibility ${ }^{14}$

$$
\chi(\mathbf{q})=\frac{\chi_{0}(\mathbf{q})}{1+[U / 2+V(\mathbf{q})] \chi_{0}(\mathbf{q})}
$$



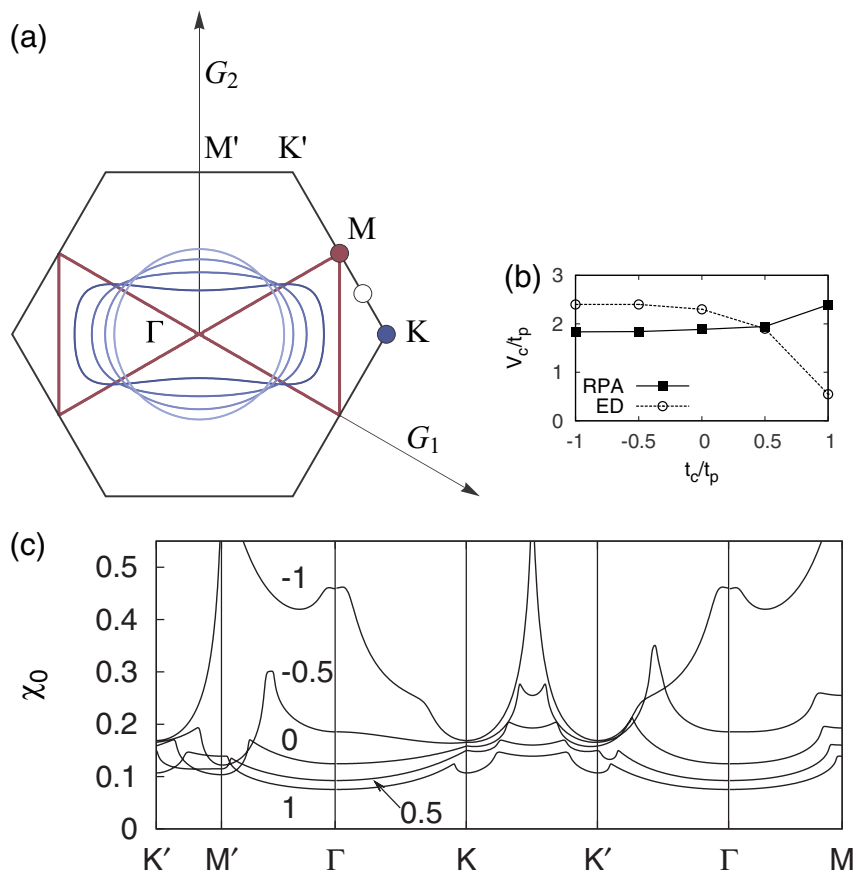

FIG. 11. (Color online) (a) Brillouin zone of the triangular lattice (black), reciprocal lattice vectors $G_{1}=(2 \pi,-2 \pi / \sqrt{3})$ and $G_{2}=$ $(0,4 \pi / \sqrt{3})$ (arrows), and Fermi surface: from light blue to dark blue, $t_{c} / t_{p}=1,0.5,0,-0.5 ;$ red, $t_{c} / t_{p}=-1$ (same color code as in Fig. 2). The blue and red dots are respectively the threefold wave vector $\mathbf{Q}=$ $\mathbf{K}=(4 \pi / 3,0)$ and the nesting wave vector $\mathbf{Q}_{F}=\mathbf{M}=(\pi, \pi / \sqrt{3})$ connecting flat segments of the Fermi surface at $t_{c} / t_{p}=-1$ (red), while the white dot is the wave vector associated with the predominant instability for $t_{c} / t_{p}=1$. (b) The critical value of the intersite Coulomb interaction $V_{3 \mathrm{CO}}$ for $U=5 t_{p}$, comparing the RPA and $\mathrm{ED}$ results. (c) Free-electron susceptibility $\chi_{0}$ along symmetry lines of the Brillouin zone, for different values of $t_{c} / t_{p}$.

at a given wave vector. Here $\chi_{0}(\mathbf{q})$ is the noninteracting susceptibility of the lattice, $V(\mathbf{q})$ is the interaction potential in Fourier space, and $U$ is the on-site repulsion. For isotropic nearest-neighbor interactions on the triangular lattice we have

$$
\begin{aligned}
V(\mathbf{q})= & 2 V\left\{\cos \left(q_{x}\right)\right. \\
& \left.+\cos \left[\left(q_{x}+\sqrt{3} q_{y}\right) / 2\right]+\cos \left[\left(q_{x}-\sqrt{3} q_{y}\right) / 2\right]\right\} .
\end{aligned}
$$

An instability occurs when the denominator in Eq. (A1) vanishes, which requires $-V(\mathbf{q})=\chi_{0}^{-1}(\mathbf{q})+U / 2$. In principle the above equation can describe both charge ordering, driven by the Coulomb iteraction $-V(\mathbf{q})$ that is maximum at the six equivalent threefold wave vectors $\mathbf{Q}=( \pm 2 \pi / 3, \pm 2 \pi / \sqrt{3})$, $( \pm 4 \pi / 3,0)$ [blue dot in Fig. 11(a)], and a charge-density wave $(\mathrm{CDW})$ induced by a large $\chi_{0}$. The evolution of the free-electron susceptibility with frustration is illustrated in Fig. 11(b).

A CDW instability occurs for $t_{c} / t_{p}=-1$ due to a perfect nesting between parallel segments of the Fermi surface [red lines in Fig. 11(a)], at wave vector $\mathbf{Q}_{F}=(\pi, \pi / \sqrt{3})$ (red dot, corresponding to the $\mathbf{M}$ point), but is washed out at $U / t_{p} \gtrsim 7$, where the threefold order is always favored. An instability also appears to compete with the $3 \mathrm{CO}$ in the case $t_{c} / t_{p}=1$ for $U \lesssim 2 t_{p}$, at a wave vector $\mathbf{Q}_{1}=(2.62,2.72)$ (white dot). Such vector lies at the intersection between a circle of radius $\left|\mathbf{Q}_{1}\right|=$ $2 k_{F}$ and the boundary of the Brillouin zone. It represents a compromise between a Fermi surface instability and a genuine charge ordering, as it benefits from both a large $\chi_{0}$ and a large $-V(\mathbf{q})$. For values of the frustration ratio $\left|t_{c} / t_{p}\right| \lesssim 0.9$, the RPA predicts that the $3 \mathrm{CO}$ transition is dominant for all $U>0$.

The critical coupling $V_{3 \mathrm{CO}}$ at the threefold instability is shown as a function of the geometrical frustration in Fig. 11(b). The RPA predicts an increase of $V_{3 \mathrm{CO}}$ with $t_{c} / t_{p}$ which originates from a decrease of the density of states at the Fermi level. ${ }^{56}$ We see that even at a relatively low value of $U=5 t_{p}$, the RPA result does not agree with the exact diagonalization data, showing an opposite trend for positive $t_{c} / t_{p}$ ratios. In the ED, the $t_{c}$ dependence is governed by the stabilization of the pinball-liquid phase, which is not captured by the weak-coupling RPA argument.

\section{APPENDIX B: MEAN-FIELD POTENTIAL ENERGY IN THE CHARGE-ORDERED STATE}

The Hartree expression for the potential energy per site in a charge-ordered state with threefold symmetry reads

$$
\begin{aligned}
E_{H}= & \frac{U}{3}\left(n_{A \uparrow} n_{A \downarrow}+n_{B \uparrow} n_{B \downarrow}+n_{C \uparrow} n_{C \downarrow}\right) \\
& +V\left(n_{A} n_{B}+n_{A} n_{C}+n_{B} n_{C}\right) .
\end{aligned}
$$

We take $n_{A \uparrow}=n_{A \downarrow}=n_{B \uparrow}=n_{B \downarrow}=1, n_{C \uparrow}=1 / 2, n_{C \downarrow}=0$ corresponding to the Hartree solution for the $3 \mathrm{CO}$ state and $n_{A \uparrow}=n_{B \uparrow}=3 / 4, n_{A \downarrow}=n_{B \downarrow}=1, n_{C \uparrow}=1, n_{C \downarrow}=0$ for the pinball-liquid phase (see Fig. 4). Inserting these values in the preceding expression we find

$$
\begin{gathered}
E_{H}^{(M)}=(9 / 16) U+(27 / 4) V, \quad \mathrm{HM} ; \\
E_{H}^{(\mathrm{PL})}=(1 / 2) U+(13 / 2+1 / 16) V, \quad \mathrm{PL} ; \\
E_{H}^{(3 \mathrm{CO})}=(2 / 3) U+6 V, \quad 3 \mathrm{CO} .
\end{gathered}
$$

The potential energy calculated by ED closely follows the $V$ dependence predicted by the above mean-field equations (not shown). The transition from the pinball state to the threefold CO state is well captured by the mean-field analysis: The ED data closely follow the value $V_{c}=U / 3$ obtained by equating $E_{H}^{(\mathrm{PL})}=E_{H}^{(3 \mathrm{CO})}$.
${ }^{1}$ V. J. Emery and S. A. Kivelson, Phys. Rev. Lett. 74, 3253 (1995).

${ }^{2}$ J. Merino and R. H. McKenzie, Phys. Rev. B 61, 7996 (2000).

${ }^{3}$ P. B. Allen, Physica B 318, 24 (2002).

${ }^{4}$ N. E. Hussey, K. Takenaka, and H. Takagi, Philos. Mag. 84, 2847 (2004)

${ }^{5}$ M. Calandra and O. Gunnarsson, Phys. Rev. B 66, 205105 (2002).
${ }^{6}$ O. Gunnarsson, M. Calandra, and J. E. Han, Rev. Mod. Phys. 75, 1085 (2003).

${ }^{7}$ D. N. Basov and A. V. Chubukov, Nature Phys. 7, 272 (2011).

${ }^{8}$ K. Miyagawa, A. Kawamoto, and K. Kanoda, Phys. Rev. B 61, R7679 (2000). 
${ }^{9}$ K. Yamamoto, K. Yakushi, K. Miyagawa, K. Kanoda, and A. Kawamoto, Phys. Rev. B 65, 085110 (2002).

${ }^{10}$ M. Watanabe, Y. Nogami, K. Oshima, H. Mori, and S. Tanaka, J. Phys. Soc. Jpn. 68, 2354 (1999).

${ }^{11}$ M. Watanabe, Y. Noda, Y. Nogami, and H. Mori, J. Phys. Soc. Jpn. 73, 116 (2004).

${ }^{12}$ M. Watanabe, Y. Noda, Y. Nogami, and H. Mori, J. Phys. Soc. Jpn. 74, 2011 (2005).

${ }^{13}$ T. Mori, Bull. Chem. Soc. Jpn. 73, 2243 (2000).

${ }^{14}$ J. Merino, A. Greco, N. Drichko, and M. Dressel, Phys. Rev. Lett. 96, 216402 (2006).

${ }^{15}$ L. Cano-Cortés, J. Merino, and S. Fratini, Phys. Rev. Lett. 105, 036405 (2010).

${ }^{16}$ K. Miyake, S. Schmitt-Rink, and C. M. Varma, Phys. Rev. B 34, 6554 (1986).

${ }^{17}$ P. Coleman, Heavy Fermions: Electrons at the Edge of Magnetism, Handbook of Magnetism and Advanced Magnetic Materials, edited by Helmut Kronmuller and Stuart Parkin, Vol 1: Fundamentals and Theory (John Wiley and Sons, 2007), pp. 95-148.

${ }^{18}$ P. Gegenwart, Q. Si, and F. Steglich, Nature Phys. 4, 186 (2008).

${ }^{19}$ H. v. Löhneysen, A. Rosch, M. Vojta, and P. Wölfle, Rev. Mod. Phys. 79, 1015 (2007).

${ }^{20}$ K. Kuroki, Sci. Technol. Adv. Mater. 10, 024312 (2009).

${ }^{21}$ M. Udagawa and Y. Motome, Phys. Rev. Lett. 98, 206405 (2007).

${ }^{22}$ J. Jaklič and P. Prelovšek, Phys. Rev. B 49, 5065 (1994).

${ }^{23}$ A. Liebsch, H. Ishida, and J. Merino, Phys. Rev. B 78, 165123 (2008).

${ }^{24}$ A. Liebsch, H. Ishida, and J. Merino, Phys. Rev. B 79, 195108 (2009).

${ }^{25}$ H. Seo, C. Hotta, and H. Fukuyama, Chem. Rev. 104, 5005 (2004).

${ }^{26}$ We work explicitly with electrons. To make contact with other theoretical works in the literature, the case $t_{p}<0$ for $n=3 / 2$ is equivalent to taking $t_{p}>0$ for $n=1 / 2$ (one-quarter filling) without changing the absolute sign of $t_{c}$.

${ }^{27}$ H. Seo, J. Phys. Soc. Jpn. 69, 805 (2000).

${ }^{28}$ M. Kaneko and M. Ogata, J. Phys. Soc. Jpn. 75, 014710 (2006).

${ }^{29}$ K. Kuroki, J. Phys. Soc. Jpn. 75, 114716 (2006).

${ }^{30}$ R. T. Clay, S. Mazumdar, and D. K. Campbell, J. Phys. Soc. Jpn. 71, 1816 (2002).

${ }^{31}$ J. Merino, H. Seo, and M. Ogata, Phys. Rev. B 71, 125111 (2005).

${ }^{32}$ S. Nishimoto, M. Shingai, and Y. Ohta, Phys. Rev. B 78, 035113 (2008).

${ }^{33}$ T. Mori, J. Phys. Soc. Jpn. 72, 1469 (2003).

${ }^{34}$ C. Hotta and N. Furukawa, Phys. Rev. B 74, 193107 (2006).

${ }^{35}$ M. Miyazaki, C. Hotta, S. Miyahara, K. Matsuda, and N. Furukawa, J. Phys. Soc. Jpn. 78, 014707 (2009).

${ }^{36}$ H. Watanabe and M. Ogata, J. Phys. Soc. Jpn. 75, 063702 (2006).

${ }^{37}$ In this work we consider the maximally frustrated case concerning interactions $\left(V_{p}=V_{c}=V\right)$, while varying the degree of frustration in the electron motion via the parameter $t_{c} / t_{p}$. Previous studies of the EHM on the square lattice correspond, in the present language, to the case $V_{p}=V$ and $V_{c}=0$.

${ }^{38}$ H. Mori, S. Tanaka, and T. Mori, Phys. Rev. B 57, 12023 (1998).

${ }^{39}$ R. H. McKenzie et al., Phys. Rev. B 64, 085109 (2001).

${ }^{40}$ F. Nad, P. Monceau, and H. M. Yamamoto, Phys. Rev. B 76, 205101 (2007).
${ }^{41}$ M. Tamura, H. Kuroda, S. Uji, H. Aoki, M. Tokumoto, A. G. Swanson, J. S. Brooks, C. C. Agosta, and S. T. Hannahs, J. Phys. Soc. Jpn. 63, 615 (1994).

${ }^{42}$ L. Cano-Cortés et al., Eur. Phys. J. B 56, 173 (2007).

${ }^{43}$ E. Scriven and B. J. Powell, J. Chem. Phys. 130, 104508 (2009).

${ }^{44}$ H. Seo et al., J. Phys. Soc. Jpn. 75, 051009 (2006); T. Mori, H. Mori, and S. Tanaka, Bull. Chem. Soc. Jpn. 72, 179 (1999).

${ }^{45}$ A. J. Millis, in Optical Conductivity and Correlated Electron Physics, in Strong Interactions in Low Dimensions, edited by D. Baeriswyl and L. DeGiorgi (Springer Verlag, Berlin, 2004).

${ }^{46}$ M. M. Qazilbash, J. J. Hamlin, R. E. Baumbach, L. Zhang, D. J. Singh, M. B. Maple, and D. N. Basov, Nature Phys. 5, 647 (2009).

${ }^{47}$ P. F. Maldague, Phys. Rev. B 16, 2437 (1977).

${ }^{48}$ G. Seibold and E. Sigmund, Z. Phys. B 101, 405 (1996).

${ }^{49} \mathrm{~A}$ word of caution is needed here since due to accidental degeneracies of the noninteracting kinetic energy, $K_{0}$ is independent of $t_{c} / t_{p}$ in the small $N_{c}=12$ cluster used which coincides with the $t_{c}=0$ kinetic energy of the extended tight-binding model of the lattice. At $U>5 t$ degeneracies are split and dependence on $t_{c} / t_{p}$ is recovered as expected.

${ }^{50}$ C. Hotta, N. Furukawa, A. Nakagawa, and K. Kubo, J. Phys. Soc. Jpn. 75, 123704 (2006).

${ }^{51}$ A. Ralko et al., Phys. Rev. B 74, 134301 (2006).

${ }^{52}$ A. Uhlmann, Rep. Math. Phys. 9, 273 (1976).

${ }^{53}$ M. A. Nielsen and I. L. Chuang, Quantum Computation and Quantum Information (Cambridge University Press, Cambridge, 2000).

${ }^{54}$ L. Campos Venuti and P. Zanardi, Phys. Rev. Lett. 99, 095701 (2007).

${ }^{55}$ P. Buonsante and A. Vezzani, Phys. Rev. Lett. 98, 110601 (2007). ${ }^{56}$ S. Nishimoto and C. Hotta, Phys. Rev. B 79, 195124 (2009).

${ }^{57}$ J. A. Hertz, Phys. Rev. B 14, 1165 (1976).

${ }^{58}$ A. J. Millis, Phys. Rev. B 48, 7183 (1993).

${ }^{59}$ B. L. Altshuler, L. B. Ioffe, and A. J. Millis, Phys. Rev. B 52, 5563 (1995).

${ }^{60}$ A. Rosch et al., Phys. Rev. Lett. 79, 159 (1997).

${ }^{61}$ R. Hlubina and T. M. Rice, Phys. Rev. B 51, 9253 (1995).

${ }^{62}$ A. Rosch, Phys. Rev. Lett. 82, 4280 (1999).

${ }^{63}$ T. Ishiguro, K. Yamaji, and G. Saito, Organic Superconductors, 2nd ed. (Springer, New York, 2001).

${ }^{64}$ S. Yasuzuka, K. Kobayashi, H. Nishikawa, H. Yoshino, and K. Murata, J. Phys. Soc. Jpn. 75, 083710 (2006).

${ }^{65}$ Y. Weng, H. Yoshino, N. Hiratani, H. Akutsu, J. Yamada, K. Kikuchi, and K. Murata, Synth. Met. 159, 2394 (2009).

${ }^{66}$ K. Takenaka, M. Tamura, N. Tajima, and H. Takagi, Phys. Rev. Lett. 95, 227801 (2005).

${ }^{67}$ J. Merino and R. H. McKenzie, Phys. Rev. Lett. 87, 237002 (2001).

${ }^{68}$ H. Mori, J. Phys. Soc. Jpn. 75, 051003 (2006).

${ }^{69}$ M. Jourdan, M. Huth, and H. Adrian, Nature (London) 398, 47 (1999).

${ }^{70}$ D. J. Scalapino, E. Loh Jr., and J. E. Hirsch, Phys. Rev. B 35, 6694 (1987).

${ }^{71}$ Y. Tanaka, Y. Yanase, and M. Ogata, J. Phys. Soc. Jpn. 73, 319 (2004).

${ }^{72}$ G. Pascut et al., Phys. Rev. Lett. 106, 157206 (2011).

${ }^{73}$ I. Mazin et al., Phys. Rev. Lett. 98, 176406 (2007). 